\title{
EQUAÇAO DE DIFERENÇA COM RETARDAMENTO DEPENDENDO DO TEMPO: \\ CASO MATRICIAL
}

\section{Lizete M. Crnkowise Fernandes Garcia}

ORIENTADOR. Pro6. Dr. Cerino Ewerton de Avellar

Dissertação apresentada ao Instituto
de Ciências Matemâticas de são Car-
los, da Universidade de são Paulo,
para a obtenção do Tîtulo de Mestre
em Matemática.


A José Paulo, aos meus pais: Catharina e Francisco e à tia cota.

D E D I C O 


\section{AGRADECIMENTOS}

Ao meu Orientador, Prof. Dr. Cerino Ewerton de Avellar, pela assistência e atenção dadas durante a realização deste trabalho.

Aos professores do Departamento de Matemática, da Universidade Federal de são Carlos e do ICMSC-USP-São Carlos.

A minha família, pelo incentivo que me deram durante todos esses anos.

Ao meu marido, Josê Paulo, pelo constante apoio.

A Sandra Lủcia de Oliveira, por sua eficiência na confecção deste trabalho.

Este trabalho foi
parcialmente fi-
nanciado pelo
CNPq $P$ PICD.




\section{SUMMARY}

On this work we study stability properties of a system of Difference Equations $\quad x(t)=\sum_{k=1}^{N} A_{k} x\left(t-r_{k}\right)$, $\mathbf{x} \in \mathbf{R}^{\mathrm{n}}, \mathrm{A}_{\mathrm{k}^{\prime}} \mathrm{n \times n}$ constant matrices, $\mathrm{r}_{\mathrm{k}}>0$, in the case that we allow the delays $r_{k}$ to vary in time.

The main result we obtained is that if the above system is stable and the matrices $A_{k}$ satisfy a commutative condiction $A_{k} A_{j}^{*}=A_{j}^{*} A_{k^{\prime}}, j, k=1,2, \ldots, n$, then the system $x(t)=\sum_{k=1}^{N} A_{k} x\left(t-r_{k}(t)\right)$ is also stable. 
Pāgina

INTROOUÇAO

CAPITULO I

Definições e Resultados Bâsicos ................... 04

1. Vetores, Matrizes e Normas .................... 04

2. Operadores de Diferença ...................... 10

2.1. Operadores de Diferença

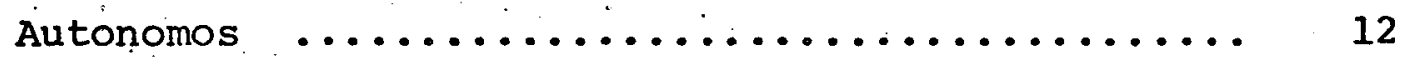

3. Medidas de não compacidade em $c([-r, \infty), p(t)) \ldots \ldots . .24$

CAPITULO II

Equações de Diferença com Retardamento Dependendo do Tempo: Caso Matricial 


\section{INTRODUÇAO}

Uma classe importante das equações diferenciais fun cionais são as chamadas "equações diferenciais do tipo neu tro" que são, a grosso modo, equações cuja função incógnita e suas derivaảas, dependem não só do instante presente como tam bém do passado.

Um modelo simples para tais equações é do tipo

$$
\dot{x}(t)-c \dot{x}(t-r)=a x(t)+b x(t-r)+f(t)
$$

onde $\mathrm{a}, \mathrm{b}, \mathrm{c}$ e $\mathrm{r}$ são constantes, $\mathrm{r}>0, \mathrm{c} \neq 0$ e $\mathrm{f}$ uma função real contínua.

Para uma equação do tipo (1), o conceito de solu ção e o apropriado espaço de condições iniciais fica, em ge ral difícil de se descrever (ver Hale [08], capitulo 1). uma maneira de contornar esse problema foi iniciada com Hale e Meyer [09] e consiste em reescrever a equação (I) na forma

$$
\frac{d}{d t}[x(t)-c x(t-r)]=a x(t)+b x(t-r)+f(t)
$$

desenvolvendo uma teoria para as chamadas "equações diferen ciais de diferença, do tipo neutro" que de uma maneira geral se escreve

$$
\frac{d}{d t} D x_{t}=f\left(t, x_{t}\right)
$$

onde D, chamado "operador de äiferença", é um operador line ar,continuo que será precisamente definido no capítulo I do nosso trabalho. 
No estudo da teoria de estabilidade e comportamento assintōtico das soluções dessas equações diferenciais de dife rença, do tipo neutro, a maior dificuldade reside no estudo das propriedades da "equação de diferença" associada

$$
D x_{t}=0
$$

Nos últimos anos, muitos autores, Cruz e Hale [05], Henry [10], Silkowski [13], Melvin [11], Avellar e Hale [01], Siqueira [12], Ferreira [06], tem se preocupado com o estudo das equações de diferença do tipo

$$
x(t)-\sum_{k=1}^{N} A_{k} x\left(t-r_{k}\right)=0
$$

onde $x \in \mathbf{R}^{n}, A_{k}, k=1,2, \ldots, N$, são matrizes $n \times n$ e $0<r_{k} \leq r$

Muitos problemas surgem ao se tentar estudar a es tabilidade das soluções de um sistema do tipo (5), quando per mitimos variações nos coeficientes $A_{k}$ e nos retardamentos $\mathbf{r}_{\mathrm{k}}$ que, em geral, são parâmetros que não são conhecidos prẹ cisamente.

Melvin [1I] estabeleceu uma condição necessāria e suficiente para se garantir "estabilidade nos retardamentos". de (5), no caso escalar. Silkowski [13] estendeu esse resultạ do para o caso matricial, conforme veremos no capitulo I. Ultimamente tem-se estudado o problema de estabili dade de equações de diferença da forma

$$
x(t)-\sum_{k=1}^{N} A_{k}(t) x\left(t-r_{k}(t)\right)=0
$$


onde os coeficientes $A_{k}(t) \quad e$ os retaräamentos $r_{k}(t)$, $k=1,2, \ldots, N$, são funções do tempo.

Siqueira [12], em sua dissertação de mestrado, 're solveu o problema para o caso escalar $A_{k}(t) \in \mathbf{R}, x(t) \in \mathbf{R}$.

o objetivo de nosso trabalho é estudar a estabilida de das soluções de (5) no caso matricial com os retardamentos variando no tempo. Utilizaremos, basicamente, a mesma tēc nica usada por siqueira [12], introduzindo uma condição de comutatividade nos coeficientes $A_{k}$. 
CAPITULO I

Definições e Resultàdos Básicos

Neste capítulo faremos uma revisão e resumo de al guns tópicos que são fundamentais para o desenvolvimento do nosso trabalho [14].

\section{Vetores, Matrizes e Normas}

Seja $E^{\mathrm{n}}$ o espaço vetorial complexo de dimensão $n$ dos vetores colunas $\mathrm{x}$, denotados por

$$
x=\operatorname{col}\left(x_{1}, x_{2}, \ldots, x_{n}\right)=\left[\begin{array}{c}
x_{1} \\
x_{2} \\
\vdots \\
x_{n}
\end{array}\right]
$$

seu transposto $x^{T}=\left(x_{1}, x_{2}, \ldots, x_{n}\right)$ e seu transposto conju gado $x^{*}=\left(\bar{x}_{1}, \bar{x}_{2}, \ldots, \bar{x}_{n}\right)$.

$$
\text { Seja } x=\operatorname{col}\left(x_{1}, x_{2}, \ldots, x_{n}\right) \in E^{n}, \quad \text { indicaremos }
$$

por

$$
\|x\|=\left(x^{*} x\right)^{3 / 2}=\left(\sum_{i=1}^{n}\left|x_{i}\right|^{2}\right)^{1 / 2}
$$

a norma Euclidiana de $x$.

$$
\text { Seja } A=\left[a_{i j}\right] \text { uma matriz complexa } n \times n \text { e se }
$$
jam $\lambda_{1}, \lambda_{2}, \ldots, \lambda_{n}$, seus autovalores. Indicaremos por

$$
\gamma(A)=\operatorname{māx}_{1<i<n}\left|\lambda_{i}\right|
$$


- raio espectral da matriz $A$.

Geometricamente, o raio espectral de $A$, representa, no plano complexo, o raio do menor disco $|z| \leq R$ com cen tro na origem, que contém todos os autovalores de A.

Definição 1.1. Se $A=\left[a_{i j}\right]$ é uma matriz comple xa nxn , a norma espectral de A é definida por

$$
\|A\|=\sup _{x \neq 0} \frac{\|A x\|}{\|x\|}
$$

A norma espectral assim definida; goza de todas as propriedades usuais da norma Eucliäiana de um vetor $x$, isto é.
i). $\|A\| \geq 0,\|A\|=0 \Longleftrightarrow A=0$
i1) $\|\alpha A\|=|\alpha| \cdot\|A\|, \quad \alpha$ escalar
iii) $\|A+B\| \leq\|A\|+\|B\|$
iv) $\|A \cdot B\| \leq\|A\| \cdot\|B\|$

Além disso,

v) $\|A x\| \leq\|A\| \cdot\|x\|, \forall x \in E^{n}$

vi) Existe $y \in E^{n}, y \neq 0$, tal que $\|A y\|=\|A\| \cdot\|y\|$

Os conceitos de norma espectral e raio espectral es tão relacionados pela seguinte: 
- Proposição 1.1. Para toda matriz quadrada A temos

$\|A\| \geq Y(A)$

\section{Prova:}

Seja $\lambda$ um autovalor de $A$ e seja $: x \neq 0$ um autovetor associado a $\lambda$, isto $\overline{\mathrm{e}}, \mathrm{Ax}=\lambda \mathrm{x}$ então,

$$
|\lambda| \cdot\|x\|=\|\lambda x\|=\|A x\| \leq\|A\| \cdot\|x\|
$$

- que implica $\|A\| \geq|\lambda|$, qualquer que seja o autovalor de A, o que conclui a demonstração.

Consideraremos agora as matrizes:

$A=\left[\begin{array}{ccc}a_{11} & \cdots & a_{1 n} \\ \vdots & & \vdots \\ \vdots & \ddots & a_{n n}\end{array}\right], \quad A^{T}=\left[\begin{array}{ccc}a_{11} & \cdots & a_{n 1} \\ \vdots & & \vdots \\ a_{1 n} & \cdots & a_{n n}\end{array}\right]$ e $A^{*}=\left[\begin{array}{lll}\bar{a}_{11} & \cdots & \bar{a}_{n 1} \\ \vdots & & \vdots \\ \bar{a}_{1 n} & \cdots & \bar{a}_{n n}\end{array}\right]$

denotam, respectivamente a matriz, $n \times n, A=\left[a_{i j}\right] ;$ sua transposta $A^{T}=\left[a_{j i}\right]$ e sua transposta conjugada $A^{\star}=\left[\bar{a}_{j i}\right]$, então $:$ produto $A^{*} A$ é aincia uma matriz $n \times n$ e a nor ma espectral de $A$ pode ser expressa em termos do raio espeç tral de $A^{*} A$ de acordo com o seguinte:

Teorema 1.1. Para toda matriz quadrada $A$, temos

$$
\|A\|=\left[\gamma\left(A^{*} A\right)\right]^{1 / 2}
$$

\section{Prova:}

Como $\left(A^{*} A\right)^{*}=A^{*} A$, o que implica $A^{*} A$ hermitiana e, desde que $x^{*} A^{*} A x=(A x)^{*}(A x)=\|A x\|^{2} \geq 0, \quad \forall x \in E^{n}$, 
$A^{*} A$ é positiva definida.

Seja: $\left\{v_{i}\right\}_{i=1}^{i n}$ um conjunto ortogonal de autoveto res de $A^{*} A$, isto $e, A^{*} A v_{i}=\lambda_{i} v_{i}, \quad i=1,2, \ldots, n$, $0 \leq \lambda_{1} \leq \lambda_{2} \leq \cdots \leq \lambda_{n}, \quad v_{i}{ }^{*} v_{j}=0$ para $i \neq j \quad e$ $v_{i} * v_{j}=1$ para $i=j$.

Para todo $x \in E^{n}, \quad x \neq 0$, podemos escrever $x=\sum_{i=1}^{n} c_{i} v_{i}$, então

$\left(\frac{\|A x\|}{\|x\|}\right)^{i}=\frac{x^{*} A^{*} A x}{x^{*} x}=\frac{\sum_{i=1}^{n}\left|c_{i}\right|^{2} \lambda_{i}}{\sum_{j=1}^{n}\left|c_{j}\right|^{2}}$ oque implica $0 \leq \lambda_{1} \leq\left(\frac{\|\mathrm{Ax}\|}{\|\mathrm{x}\|}\right)^{2} \leq \lambda_{\mathrm{n}}$

Mas, para $x=v_{n}$ temos $\frac{\left\|A v_{n}\right\|}{\left\|v_{n}\right\|}=\lambda_{n}$.

Iogo,

$\|A\|^{2}=\sup _{x \neq 0}\left(\frac{\|A x\|}{\|x\|}\right)^{2}=\lambda_{n^{\prime}}=\gamma\left(A^{*} A\right)$.

Portanto,

$$
\|A\|=\left[\gamma\left(A^{*} A\right)\right]^{1 / 2}
$$


Lema 1.1. Seja A uma matriz hermitiana, nxnn, isto ê, $A=A^{*}$, então $\|A\|=\gamma(A)$.

De fato,

$$
\|A\|^{2}=\gamma\left(A^{*} A\right)=\gamma\left(A^{2}\right)
$$

Fazendo $B=A^{i}$, notamos que $B$ é hermitiana, Lo go, $A$ e $B$ são normais, isto é, $\mathrm{AA}^{*}=\mathrm{A}^{*} \mathrm{~A}$ e $\mathrm{BB}^{*}=\mathrm{B}^{*} \mathrm{~B}$.

Então, A. B B podem ser escritas na forma $A=U^{*} D U$ e $B=U^{*} D^{2} U$, onde $U$ é unitária e $D$ é diago nal:

Se $F(D)=\left\{\lambda_{1}, \dot{\lambda}_{2}, \ldots, \lambda_{n}\right\}$ denota o conjunto de autovalores de $D$, então $F\left(D^{i}\right)=\left\{\lambda_{1}^{2}, \lambda_{2}^{2}, \ldots, \lambda_{n}^{2}\right\}$ de notará o conjunto de autovalores de $\mathrm{D}^{2}$.

Temos que

$$
\begin{aligned}
& \gamma\left(D^{2}\right)=\operatorname{maxx}_{1 \leq i \leq n}\left|\lambda_{i}^{2}\right|=\left|\lambda_{k}^{2}\right|=\left|\lambda_{k}\right|^{2}=\gamma^{2}(D) \quad \text { o que implica em } \\
& \gamma\left(A^{2}\right)=\gamma^{2}(A) .
\end{aligned}
$$$$
\text { Logo, }\|A\|=\gamma(A)
$$

Um outro caso particular interessante é dado pelo:

Corolário 1.1. Seja A uma matriz normal nxn isto é, $A A^{*}=A^{*} A$. Se $\|A\|$ é a norma espectral de $A$, então $\|A\|=\gamma(A)$. 
Entretanto, esse fato não é verdadeiro em geral. Daremos um exemplo em que $\|A\|>\gamma(A)$.

\section{Exemplo 1.1.}

$$
\text { Seja a matriz } A=\left[\begin{array}{cc}
1 / 2 & 1 \\
0 & 1 / 2
\end{array}\right] \text { cujos autovalores }
$$

são $\lambda_{1}=\lambda_{2}=\frac{1}{2}$, o que implica $\gamma(A)=\frac{1}{2}$

$$
\text { Por outro lado, os autovalores de } A^{*} A=\left[\begin{array}{cc}
1 / 4 & 1 / 2 \\
1 / 2 & 5 / 4
\end{array}\right]
$$

são:

$$
\lambda_{1}=(3+2 \sqrt{2}) / 4 \text { e } \quad \lambda_{2}=(3-2 \sqrt{2}) / 4
$$

Portanto, $\gamma\left(A^{*} A\right)=(3+2 \sqrt{2}) / 4$.

\section{Assim,}

$$
\begin{aligned}
& \|A\|=\left[\gamma\left(A^{*} A\right)\right]^{1 / 2}=[(3+2 \sqrt{2}) / 4]^{1 / 2}>\frac{1}{2}=\gamma(A) \text { ou seja, } \\
& \|A\|=\gamma(A)
\end{aligned}
$$




\section{Operadores de Diferença}

Neste parăgrafo, relataremos definições e propriedades relacionadas com os chamados operadores de diferença. Maiores detalhes podem ser encontrados em Hale [08] capitulo 12.

Seja $\mathbf{R}^{\mathrm{n}}$ o espaço vetorial normado $\mathrm{n}$-dimensional, com norma $||,. r>0$ um número real dado.

Denotamos por $C=C\left([-r, 0], \mathbf{R}^{n}\right)$ o espaço das funções continuas do intervalo $[-r, 0]$ em $\mathbf{R}^{\mathbf{n}} \operatorname{com}\|\phi\|$, $\phi \in C$, definida por $\|\phi\|=\sup _{-r \leq \theta \leq 0}|\phi(\theta)|$.

Se $x$ è uma função contínua $x:[\sigma-r, \sigma+a) \rightarrow \mathbb{R}^{\mathbf{n}}$ então para cada $t, \sigma \leq t<\sigma+a, \cdot x_{t} \in C$ é defìnida por $x_{t}(\theta)=x(t+\theta), \quad-r \leq \theta \leq 0$.

Seja $\Omega$ um subconjunto de $\mathbf{R} \times \mathbf{C}$ e seja $D: \Omega \rightarrow \mathbb{R}^{\mathbf{n}}$ um operador continuo $D(t, \phi)$, iinear em $\phi$. Pelo Teorema de Representação de Riesz, existe uma matriz $n(t, \theta)$, nxn, de variação limitada em $\theta \in[-r, 0]$ tal que

$$
D(t, \phi)=\int_{-r}^{0}\left[d_{\theta} n(t, \theta)\right] \phi(\theta)
$$

onde estamos subentendendo que estendemos a definição de $n(t, \theta)$ para $\theta \in \mathbf{R}$, colocando

$$
\begin{array}{lll}
n(t, \theta)=n(t,-r) & \text { para } & \theta \leq-r \\
n(t, \theta)=n(t, 0) & \text { para } & \theta \geq 0
\end{array}
$$


Dizemos que $\underline{D(t, \phi)}$ é atômico no zero, sobre $\Omega$,

se:

(i) A matriz $A(t)=n\left(t, 0^{+}\right)-n\left(t, 0^{-}\right)$é continua e inversivel para todo $t \in \mathbf{R}$.

(ii) Existe uma função escalar, $\gamma(t, s)$, contínua em $t \in R, s \geq 0$, com $\gamma(t, 0)=0$ tal que $\left|\int_{-s}^{0-}\left[d_{\theta} n(t, \theta)\right] \phi(\theta)-A(t) \phi(0)\right| \leq \gamma(t, s)\|\phi\|$

Exemp 10 1.2.

Se $D \phi=\phi(0)$ para todo $\phi$, então $D$ é atômico no zero.

\section{Exemplo 1. 3.}

Se $D \phi=\phi(0)+B \phi(-r)$, onde $r>0$ e B è uma matriz constante $\mathrm{nxn}$, então: D é atômico no zero.

Consideremos a "equação de diferença" homogênea

(1.1) $\left\{\begin{array}{lc}D\left(t, x_{t}\right)=0, & t \geq 0 \\ x(t)=\phi(t), & -r \leq t \leq 0\end{array}\right.$

onde $\quad D(t, \phi)$ linear e atômico no zero.

Definimos solução de (1.1) como sendo função contínua $x \in C\left([-r, a), R^{n}\right), a \leq+\infty$ tal que $\left(t, x_{t}\right) \in \Omega$ para $\dot{0} \leq t<a, D\left(t, x_{t}\right)=0,0 \leq t<a$ e $x_{0}=\phi$.

Assim, para que essa definição faça sentido, temos que restringir o espaço de condições iniciais tomando 


$$
C_{D}=\{\phi \in C: D(0, \phi)=0\}
$$

\subsection{Operadores de Diferença Autonomos}

Seja $D: C \rightarrow \mathbb{R}^{n}$, linear, continuo e atômico no zero, representado pela integral de stieltjes:

$$
D \phi=\phi(0)-\int_{-\mathbf{r}}^{0}[d \mu(\theta)] \phi(\theta)
$$

onde $\mu$ é uma matriz de variação limitada que se anula no zero, contínua pela esquerda em $[-r, 0]$ e $\operatorname{var}_{[-\varepsilon ; 0]} \mu \rightarrow 0$ quan do $\varepsilon \rightarrow 0$

No que se segue, faremos uma restrição adicional em $D_{1}$ supondo que $\mu$ não possua parte singular, isto é, que existam uma matriz nxn integrāvel $A$ e matrizes constantes $A_{k}, n x n$, tal que

(1.2) $D \phi=D_{0} \phi+\int_{-r}^{0} A(\theta) \phi(\theta) d \theta$

(1.3) $\quad D_{0} \phi=\phi(0)-\sum_{k=1}^{N} A_{k} \phi\left(-r_{k}\right)$

onde $0<r_{k} \leq r$ e $\left.\right|^{0}|A(\theta)| d \theta<\infty$ 
. Consideremos a equação de diferença autônoma

(I.4) $\begin{cases}\mathrm{Dx}_{t}=0, & t \geq 0 \\ \mathrm{x}_{0}=\phi, & \phi \in \mathrm{C}_{D}\end{cases}$

Tal equação define um semigrupo de operadores lineares, fortemente continuos, $T_{D}(t): C_{D} \rightarrow C_{D^{\prime}} t \geq 0$, onde $T_{D}(t) \phi=x_{t}(\phi), t \geq 0, \phi \in C_{D}$ e $x_{t}(\phi)$ a solução de (1.4) Seja a a ordem do semigrupo $T_{D}(t)$, isto é,

$$
a_{D}=\inf \left\{a \in R: a \cdot k=k(a):\left\|T_{D}(t)\right\| \leq k e^{a t}, t \geq 0\right\} .
$$$$
\text { Henry [09], mostrou que. }
$$$$
a_{D}=\sup \left\{\operatorname{Re} \lambda: \operatorname{det} \Delta_{D}(\lambda)=0\right\} \text { onde }
$$$$
\Delta_{D}(\lambda)=I-\sum_{k=1}^{N} A_{k} e^{-\lambda r_{k}}-\int_{-r}^{0} e^{\lambda \theta} A(\theta) d \theta
$$

Definição 1.2. Suponhamos $D: C \rightarrow \mathbf{R}^{\mathrm{n}}$, linear, contínuo, atômico no zero. O operador $D$ é dito estável se a solução nula da equação de diferença homogênea

(1.5) $\begin{cases}D x_{t}=0, & t \geq 0 \\ x_{0}=\phi, & \phi \in C_{D}\end{cases}$

for uniformemente assintoticamente estável, isto é, para todo $\varepsilon>0$, existe $\delta=\delta(\varepsilon)>0$, tal que $\|\phi\|<\delta \Rightarrow$ 
$\Rightarrow\left\|x_{t}(\phi)\right\|<\varepsilon$, para todo $t \geq 0$, e existe $\delta_{0}>0$ tal que $\|\phi\|<\delta_{0} \Rightarrow x(t, \phi) \rightarrow 0$ quando $t \rightarrow \infty$, onde $x(t, \phi)$ é a solução de (1.5).

Teorema 1.2. (Henry) As seguintes afirmações são equivalentes:

(i) D é éstāvel

(ii) $a_{D}<0$

(iii) Existe $\delta>0$ tal que todas as soluções da equação característica associada a (1.5),

$$
\begin{aligned}
& \operatorname{det}\left[I-\sum_{k=1}^{N} A_{k} e^{-\lambda r}-\int_{-r}^{0} e^{\lambda \theta} A(\theta) d \theta\right]=0 \\
& \text { satisfazem } \operatorname{Re} \lambda \leq-\delta
\end{aligned}
$$

Exemplo 1.4.

- O operador $D \phi=\phi(0)-A \phi(-r)$, onde $\mathrm{A}$ é uma matriz $\mathrm{nxn}$, tal que todos os seus autovalores tem módulo menor que $I_{\text {; }}$ é estável.

De fato,

A equação característica

$\operatorname{det}\left[I-A e^{-\lambda r}\right]=0$

é equivalente a $\operatorname{det}[\rho I-A]=0$, onde $\rho=e^{\lambda r} . \quad$ Assim, 
$|\rho|<1$ implica $\left|e^{\lambda r}\right|=e^{\operatorname{Re} \lambda}<1 \Longleftrightarrow \operatorname{Re} \lambda \leq-\delta<0$, para algum $\delta>0$.

\section{Exemplo 1.5.}

O operador $D_{\phi} \phi=\phi(0)+\frac{1}{2} \phi(-1)+\frac{1}{2} \phi(-2)$, é estável.

De fato,

A equação característica

$$
2 e^{2 \lambda}+e^{\lambda}+1=0 \Rightarrow\left|e^{\lambda}\right|=\frac{1}{\sqrt{2}} \Longrightarrow
$$

$\Longrightarrow \operatorname{Re} \lambda=\ln \frac{1}{\sqrt{2}}<0$

Analisaremos agora, como a variação nos parầmetros do operador $\mathrm{D} \phi$, podem afetar sua estabilidade. Para tanto , vamos nos ater aos operadores de diferença do tipo

$$
D_{0} \phi=\phi(0)-\sum_{k=1}^{N} A_{k} \phi\left(-r_{k}\right)
$$

$A_{k}$ : matrizes constantes $n \times n, \quad 0<r_{k} \leq r, k=1,2, \ldots, N$.

Nas aplicações, os $A_{k}{ }^{\prime} s$ e os $r_{k}{ }^{\prime} s$ são parâmetros que nem sempre são conhecidos precisamente. $E$ importante, portanto, verificar qual o efeito de pequenas variações nesses parâmetros, sobre a estabilidade do operador.

com relação a variações nos coeficientes $A_{k}^{\prime} ' s, \quad 0$ problema é simples, desde que a parte real das raízes da equação característica 


$$
\operatorname{det}\left[I-\sum_{k=1}^{N} A_{k} e^{-\lambda r_{k}}\right]=0
$$

variem continuamente com os $\mathrm{A}_{\mathrm{k}}$ 's, conforme mostram Avellar e Hale [01]. De acordo com o item (iii) do Teorema 1.2., pequenas variações nos $A_{k}$ 's não destrõem a estabilidade de $D_{0}$. o problema da variação nos retardamentos $r_{k}{ }^{\prime} s \quad e ́$ mais delicado, como mostra o seguinte exemplo devido a Melvin. .

Exemplo 1.6. Consideremos a equação

$$
x(t)=-\frac{1}{2} x(t-1)-\frac{1}{2} x(t-2), \cdots \text { que è estāvel. }
$$

Consideremos agora a equação

$$
x(t)=-\frac{1}{2} x\left(t-1+\frac{1}{2 n+3}\right)-\frac{1}{2} x(t-2)
$$

E fácil verificar que $x(t)=\operatorname{sen}(2 n+3) \frac{\pi}{2} t$ é solução desta equação e esta função não tende a zero, quando $t+\infty$. Portanto, o operaãor

$$
D \phi=\phi(0)+\frac{1}{2} \phi\left(-1+\frac{1}{2 n+3}\right)+\frac{1}{2} \phi(-2)
$$

não é estável, embora, para n ‘...suficientemente grande, $1-\frac{1}{2 n+3}$ fique arbitrariamente próximo de 1 .

Esse fato justifica as definições que daremos a seguir.

Utilizaremos a notação, $D(r, A)$, para evidenciar a dependência dos retardamentos $r_{k}$ e dos coeficientes $A_{k}$ na 
representação de $D_{0} \cdot$ : Escreveremos

(1. 7)

$$
D(r, A) \phi=\phi(0)-\sum_{k=1}^{N} A_{k} \cdot \phi\left(-r_{k}\right)
$$

$\operatorname{com} r=\left(r_{1}, r_{2}, \ldots, r_{N}\right), A=\left(A_{1}, A_{2}, \ldots, A_{N}\right), A_{k}$, e $r_{k}$ definidos anteriormente.

Indicaremos por $\left(\mathbf{R}^{+}\right)^{N}$, o produto cartesiano de $\mathbf{R}^{+}=(0,+\infty)$ por si mesmo $\mathrm{N}$-vezes.

Definição 1.3. O operador $D(r, A)$ é dito estável localmente nos retardamentos, se existe uma vizinhança aberta de $r, I(r) \subseteq\left(\mathbb{R}^{+}\right)^{N}$ tal que $D(s, A)$ é estãvel para todo $s \in I(r)$.

Definição 1.4. o operador $D(r, A)$ é dito estável globalmente nos retardamentos se for estāvel para cada $r \in\left(R^{+}\right)^{N}$. Neșe caso, diremos tambēm que $D(r, A)$ é fortemente estável.

Teorema 1.3. (Silkowski). As seguintes afirmações são equivalentes:

(i) Para algum $r$ fixado, $r \in\left(\mathbb{R}^{+}\right)^{N} r=\left(r_{1}, \ldots, r_{N}\right)$ com $r_{k}>0$, racionalmente independentes, $D(r, A)$ é estável. 
(ii) $\gamma_{0}(A)=\sup \left\{\gamma\left(\sum_{k=1}^{N} A_{k} e^{i \theta_{k}}\right): \theta_{k} \in[0,2 \pi], k=1,2, \ldots, N\right\}<1$ onde $\gamma(B)^{\circ}$ denota $\circ$ raio espectral de uma matriz $B$ :

(iii) $D(r, A)$ é estável localmente nos retardamentos, relativamente a uma família de retardamentos.

(iv) $D(r, A)$ é estável globalmente nos retardamentos.

o resultado abaixo é devido à Melvin.

Corolário 1.1.

Se cada $A_{k}$ é escalar então. $D(r, A)$ è estável localmente nos retardamentos, se, e somente se,

$$
\sum_{k=1}^{N}\left|A_{k}\right|<1
$$

Exemplo 1.7. Consideremos a equação

$$
\begin{aligned}
& x(t)=\frac{1}{2} x(t-1)+\frac{1}{3} x(t-2) \\
& 0 \text { operador } D \phi=\phi(0)-\frac{1}{2} \phi(-1)-\frac{1}{3} \phi(-2) \text { è está- }
\end{aligned}
$$

vel localmente nos retardamentos.

De fato, 
- Exemplo 1.8. Consideremos a equação

$$
x(t)=A x(t-r)+B x(t-s), \forall r, s \in R
$$

onde $\quad A=\left[\begin{array}{rr}\alpha & 0 \\ 0 & -\alpha\end{array}\right] \quad$ e $\quad B=\left[\begin{array}{rr}0 & \alpha \\ -\alpha & 0\end{array}\right]$,

$\operatorname{com} \alpha \in \mathbf{R}, \alpha<\frac{1}{\sqrt{2}}$

$$
\text { o - operador }
$$

$$
D \phi=\phi(0)-A \phi(-r)-B \phi(-s) \text { é estável.globalmen- }
$$

te nos retardamentos.

De fato,

$$
\begin{aligned}
& A+B e^{i \theta}=\left[\begin{array}{cc}
\alpha & \alpha e^{i \theta} \\
-\alpha e^{i \theta} & -\alpha
\end{array}\right] \\
& C=A+B e^{i \theta}-\lambda I=\left[\begin{array}{cc}
\alpha-\lambda & \alpha e^{i \theta} \\
-\alpha e^{i \theta} & -\alpha-\lambda
\end{array}\right]
\end{aligned}
$$

$\operatorname{det} c=(\alpha-\lambda)(-\alpha-\lambda)+\alpha^{2} e^{2 i \theta}=-\alpha^{2}+\lambda^{2}+\alpha^{2} e^{2 i \theta}=$

$$
=\lambda^{2}-\alpha^{2}\left(1-e^{2 i \theta}\right)
$$


$\operatorname{det} c=0 \Longleftrightarrow \lambda= \pm \alpha \sqrt{1-e^{2 i \theta}}$

Queremos o supremo de $|\lambda(\theta)|$, para $\theta \in[0,2 \pi]$.

$\lambda^{2}(\theta)=\alpha^{2}[(1-\cos 2 \theta)-i \operatorname{sen} 2 \theta]$

$\left|\lambda^{2}(\theta)\right|=\sqrt{\alpha^{4}\left[(1-\cos 2 \theta)^{2}+\operatorname{sen}^{2} 2 \theta\right]}=\sqrt{\alpha^{4}(2-2 \cos 2 \theta)}=$ $=\alpha^{2} \sqrt{2-2 \cos 2 \theta}$

Assim, para $\theta \in[0,2 \pi], \quad \sup |\lambda(\theta)|^{2}=2 \alpha^{2}$ e $[0,2 \pi]$

$\therefore \quad \gamma_{O}(A, B)=\sup _{\theta \in[0,2 \pi]}|\lambda(\theta)|=\alpha \sqrt{2}$

Como $\alpha<\frac{1}{\sqrt{2}}$, temos $\gamma_{0}(A, B)<1$, e pelo Teorema (1.3), vemos que o operador $D \phi$ é estável globalmente nos retardamentos.

o seguinte resultado em relação a estabilidade de operadores $D$ com os retardamentos $r_{k}$ 's e os coeficientes $a_{k}{ }^{\prime} s$ dependendo do tempo, no caso escalar, é devido a Siqueira [12].

Teorema 1.4. Consideremos o sistema

(1.8) $\left\{\begin{array}{l}z(t)=\sum_{k=1}^{N} a_{k} z\left(t-r_{k}\right) ; \quad t \geq 0 \\ z(t)=\varphi(t) ;-r \leq t \leq 0\end{array}\right.$

onde $z(t) \in R, a_{k} e r_{k}, k=1, \ldots, N$, constantes reais, 
$0<r_{k} \leq r$, que vamos supor estāvel globalmente nos retardamentos, isto é $\sum_{k=1}^{N}\left|a_{k}\right|<1$, e consideremos o sistema pertubado:

(1.9) $\left\{\begin{array}{l}y(t)=\sum_{k=1}^{N} a_{k}(t) y\left(t-r_{k}(t)\right) ; t \geq 0 \\ y(t)=\varphi(t) ;-r \leq t \leq 0\end{array}\right.$

onde $r_{k}=[0, \infty) \rightarrow[0, \infty)$ são funções reais continuas tais que $r_{k}(0)=r_{k}, \quad r_{k}(t) \leq t+r_{k}, \quad \forall t \in[0, \infty), k=1,2, \ldots, N$; $a_{k}:[0, \infty) \rightarrow \mathbf{R}$ são funções reais continuas, tais que $a_{k}(0)=a_{k}, k=1,2, \ldots, N$ e $\sup _{t \geq 0}\left\{\sum_{k=1}^{N}\left|a_{k}(t)\right|\right\}<1$

Seja

$$
r(t)=\left\{\begin{array}{l}
\max _{1 \leq k \leq N}\left\{r_{k}(t)\right\}, t \geq 0 \\
r=\max _{1 \leq k \leq N}\left\{r_{k}\right\},-r \leq t<0
\end{array}\right.
$$

tal que $\lim _{t \rightarrow \infty}(t-r(t))=+\infty$

Então, para todo $\Psi \in C_{D^{\prime}}$ o sistema (1.9) tem uma única solução $y$ com $y(t) \rightarrow 0$, quando $t \rightarrow \infty$.

Ainda na dissertação de Siqueira [12], encontramos - seguinte contra-exemplo do caso matricial. 
Exemplo 1.9. Seja o sistema

(1.10) $\left\{\begin{array}{c}x(t)=A x(t-r)+B x(t-s), t \geq 0 \\ x(t)=\varphi(t),-a \leq t \leq 0\end{array}\right.$

onde $\quad \mathrm{x}=\left[\begin{array}{c}\mathrm{x}_{1} \\ \mathrm{x}_{2}\end{array}\right] \in \mathbf{R}^{2}, \mathrm{~A}$ e $\mathrm{B}$ matrizes constantes, $2 \mathrm{x} 2$,

onde $\quad A=\left[\begin{array}{cc}2 / 3 & 0 \\ 0 & -2 / 3\end{array}\right]$ e $B=\left[\begin{array}{cc}0 & 2 / 3 \\ -2 / 3 & 0\end{array}\right]$

$r>0, s>0, a=\max \{r, s\}$.

O operador $D(r, s)$ dado por $D(r, s) \phi=\phi(0)-A \phi(-r)-B \phi(-s)$

é estável globalmente nos retardamentos, conforme mostramos no exemplo 1.8 .

Tomando $r=r(t), s=s(t)$ funções continuas, periódicas de período 3 tal que

$$
\begin{aligned}
& r(0)=1, \quad r(1)=3, \quad r(2)=3 \\
& s(0)=2, \quad s(1)=1, \quad s(2)=1 \text { e a condição }
\end{aligned}
$$

inicial, $\varphi:[-2,0] \rightarrow \mathbf{R}^{2}, \varphi \in C_{D(r, s)}$ com

$$
\varphi(-1)=e_{1}=\left[\begin{array}{l}
1 \\
0
\end{array}\right] \quad \text { e } \quad \varphi(-2)=e_{2}=\left[\begin{array}{l}
0 \\
1
\end{array}\right]
$$

Siqueira [12], mostrou que para o sistema 
(1.11) $\left\{\begin{array}{l}x(t)=A x(t-r(t))+B x(t-s(t)), \quad t \geq 0 \\ x(t)=\varphi(t) ; \quad t \in[-2,0]\end{array}\right.$ existe uma sequência ‥ $\left\{t_{n}\right\}, t_{n} \rightarrow \infty$ quando $n \rightarrow \infty$, tal que se $x(t)$ é solução de $(1.11), x\left(t_{n}\right) \rightarrow 0$, quando $\mathrm{n} \rightarrow \infty$, isto é, o sistema (1.11) não é estável.

No nosso trabalho, daremos uma condição suficiente para se preservar a estabilidade no caso matricial, com $\because$ os retardamentos dependendo do tempo. A idéia foi generalizar o trabalho de Siqueira [12], que utiliza o Teorema do Ponto Fixo de Darbo [03], e a técnica do Teorema de Banãs [03] . 
3. Medidas de não compacidade em $C([-r, \infty), p(t)),[03],[04]$

Seja: (E, $\|$.$\| ) um espaço de Banach. para um dado$ conjunto $X$ de $E$, denotaremos por $\bar{X}$, o fecho de $X$ e por coX, a envoltória convexa de $x$.

Sejam:

$\mathrm{m}_{\mathrm{E}}$ : a familia de todos os subconjuntos limitados e não vazios de $E$.

$\mathrm{R}_{\mathrm{E}}$ : a familia de todos os subconjuntos relativamente compactos e não vazios de $E$.

Se $\dot{B}$ é uma dada familia de conjuntos, então:

${ }_{B}{ }^{C}$ : é a subfamilia de todos os conjuntos fechados de $B$.

Medida de não compacidade

Uma função $\mu: m_{E} \rightarrow[0, \infty)$ será chamada uma medida de não compacidade se satisfaz as seguintes condições:
(i) A familia
$B=\left\{x \in m_{E}: \mu(x)=0\right\}$ è não vazia e $B \subset R_{E}$.
(ii) $\mathrm{X} \subset \mathrm{Y} \Rightarrow \mu(\mathrm{X}) \leq \mu(\mathrm{Y})$
(iii) $\mu(\bar{x})=\mu(x)$
(iv) $\mu(\overline{\operatorname{cox}})=\mu(\mathrm{X})$ 


$$
\begin{aligned}
& \text { (v) } \mu^{\mu}(\lambda X+(1-\lambda) Y) \leq \lambda \mu(X)+(1-\lambda) \mu(Y) \text {, para todo } \\
& \lambda \in[0,1] \\
& \text { (vi) } \operatorname{se~} x_{n} \in m_{E}^{c}, x_{n+1} a x_{n}, n=1,2, \ldots, \text { e se } \\
& \lim _{n \rightarrow+\infty} \mu\left(x_{n}\right)=0, \text { então, o conjunto } \\
& \quad x_{\infty}=\underset{\substack{n=1 \\
n=1}}{\infty} x_{n} \neq \phi
\end{aligned}
$$

A família B na condição (i) è chamada o núcleo da medida $\mu$ e será denotada por ker $\mu$.

Temos que $(\operatorname{ker} \mu)^{\mathrm{C}}$ forma um conjunto fechado em $\mathrm{m}_{\mathrm{E}}^{\mathrm{c}}$, com relação à topologia gerada pela distâncịa de Hausdorff, E mais, a função $\mu$ é continua (e mesmo lipschitziana) com relação à distância de Hausdorff.

Se a medida de não compacidade $\mu$ satisfaz em adição, as seguintes duas condições:

$$
\begin{aligned}
& \text { (vii) } \mu(X+Y) \leq \mu(X)+\mu(Y), \forall X, Y \in E \\
& \text { (viii) } \mu(\lambda X)=|\lambda| \mu(X), \lambda \in R, \quad \forall X \in E
\end{aligned}
$$

será chamda de uma medida sublinear de não compacidade, também conhecida como medida de Kuratowski.

Seja $p(t)$, uma função contínua e definida no intervalo $[-r, \infty)$ com valores reais positivos, $\mathbf{r} \in \mathbf{R}$.

$$
\text { Denotaremos: } c([-r, \infty), p(t))=c_{p} \text { o conjunto de }
$$
todas as funções contínuas definidas no intervalo $[-r, \infty)$, tais que

$$
\sup \{|x(t)| p(t): t \geq-r\}<\infty
$$


Temos que $C_{p}$ ê um espaço de Banach com relação

a norma:

$$
\|x\|=\sup \{|x(t)| p(t): t \geq-r\}
$$

Agora, para um arbitrārio $x \in c_{p}, x \in m_{c_{p}}, \Gamma>0$, $\varepsilon .>0$, denotaremos:

$$
\begin{aligned}
& \omega^{\Gamma}(x ; \varepsilon)=\sup \{|x(t) p(t)-x(s) p(s)|: t, s e[-r, \Gamma],|t-s| \leq \varepsilon\} \\
& \omega^{\Gamma}(x, \varepsilon)=\sup \left\{\omega^{\Gamma}(x, \varepsilon): x \in X\right\} \\
& \omega_{O}^{\Gamma}(x)=\lim _{\varepsilon \rightarrow 0} \omega^{\Gamma}(x, \varepsilon) \\
& \omega_{O}(x)=\lim _{\Gamma \rightarrow \infty} \omega_{0}^{\Gamma}(x) \\
& a(x)=\lim _{\Gamma \rightarrow \infty} \sup _{x \in X}\{\sup \{|x(t)| p(t): t \geq \Gamma\}\} \\
& b(x)=\lim _{\Gamma \rightarrow \infty} \sup \{\sup \{|x(t) p(t)-x(s) p(s)|: t, s \geq \Gamma\}\}
\end{aligned}
$$

Para todo conjunto $\mathrm{x} \in \mathrm{m}_{\mathrm{C}_{\mathrm{p}}}$, denotaremos:

$$
\begin{aligned}
& \mu_{0}(x)=\omega_{0}(x)+a(x) \\
& \mu(x)=\omega_{0}(x)+b(x)
\end{aligned}
$$

Estas funções $\mu_{\mathrm{O}}(\mathrm{X})$ e $\mu(\mathrm{X})$ são medidas sublinares de não compacidade no espaço $c_{p}$.

- ker $\mu_{0}$ è a familia de todos os conjuntos 1imitados, consistindo de funções eqüicontinuas em cada inter- 
valo compacto e que $\operatorname{Iim} x(t) p(t)=0$, uniformemente com relação a $x \in X$, e ker $\mu$ ẽ a família de todos os conjuntos limitados consistindo de funções eqüicontinuas em cada intervalo compacto e tal que

$\lim _{\Gamma \rightarrow \infty}\{\sup [|x(t) p(t)-x(s) p(s)|: t, s \geq \Gamma]\}=0, \quad$ uniformemente com relação a $\mathrm{x} \in \mathrm{x}$.

Seja, M C E, conjunto não vazio e $\mu$ : medida de não compacidade em $E$.

Contração com relação a $\mu$.

Uma transformação contínua $T: M+E$, è uma contração com rèlação a $\mu$ (chamada $\mu$-contração) se:

$X \in M, X \in m_{E^{\prime}}$ então $T X \in m_{E}$ e
$\mu(T X) \leq K \mu(X)$, onde $K \in[0,1)$
Enunciaremos agora a versão modificada do Teorema do Ponto Fixo de Darbo, que utilizaremos.

Teorema 1.5. Seja C um subconjunto não vazio, limitado, convexo e fechado de um espaço de Banach E.

Seja $T: C \rightarrow C$, uma $\mu$-contração.

Intão, $T$ tem no mínimo um ponto fixo em $C$ e o conjunto $F i x T=\{x \in C$ : $T x=x\}$ pertence a ker $\mu$. 
CAPITULO II

Equações de Diferença com Retardamento Dependendo do Tempo: Caso Matricial.

Com a notação do capîtulo anterior, consideremos o sistema:

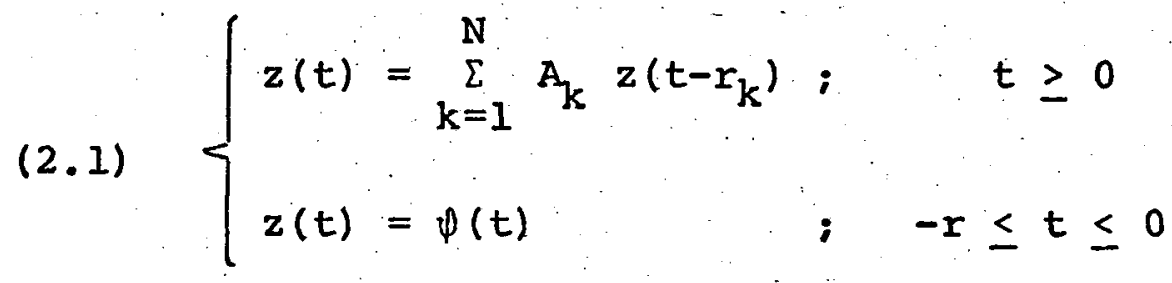

onde $z(t) \in \mathbb{R}^{n}, A_{k}$ matrizes reais $n \times n, \quad k=1,2, \ldots, N$, $0<r_{k} \leq r$, que vamos supor estável globalmente nos retardamentos, isto é, $\gamma_{0}(A)=\sup _{\theta_{k} \in[0,2 \pi]} \gamma\left(\sum_{k=1}^{N} A_{k} e^{i \theta_{k}}<1\right.$.

Associado ao sistema (2.1) temos o operador de diferença

$$
D(r, A) \phi=\phi(0)-\sum_{k=1}^{N} \overrightarrow{A_{k}} \phi\left(-r_{k}\right)
$$

Tomando a norma $\|D(r, A)\|=\underset{\left\|x_{k}\right\|_{\leq 1}}{ }\left\|\sum_{k=1}^{N} A_{k} x_{k}\right\|, \quad$ sabemos que se $\|D(r, A)\|<1$, o sistemạ $(2.1)$ é estável globalmente nos retardamentos.

Sabemos também que, no caso escalar, o sistema (2.1) é estável globalmente nos retardamentos se, e somente, se $\sum_{k=1}^{N}\left\|A_{k}\right\|<1$, isto $\bar{e},\|D(r, A)\|<1$. Entretanto, no caso 
matricial, podemos ter (2.1) estável globalmente nos retardamentos, sem que $\|D(r, A)\|$ seja menor que 1. Esse fato pode ser comprovado pelo seguinte exemplo devido a Paul Massat e que aparece em Ferreira [06].

Exemplo 2.1. Considere a equação de diferença.

$$
x(t)=A x(t-r)+B x(t-s)
$$

que com $A=\left[\begin{array}{cc}2 / 3 & 0 \\ 0 & -2 / 3\end{array}\right], \quad B=\left[\begin{array}{cc}0 & 2 / 3 \\ -2 / 3 & 0\end{array}\right], \quad e ́$ uniformemente estável globalmente nos retardamentos conforme mostramos no exemplo 1.8 .

Por outro lado, observamos que com a norma usual do $\mathbf{R}^{2}, \operatorname{temos}\left\|A \cdot\left[\begin{array}{l}1 \\ 0\end{array}\right]+B \cdot\left[\begin{array}{l}0 \\ 1\end{array}\right]\right\|=\left\|\left[\begin{array}{c}4 / 3 \\ 0\end{array}\right]\right\|=4 / 3>1$ ou seja $\|D(r, A)\|>1$. Na verdade podemos verificar nesse exemplo que, qualquer outra norma equivalente em $\mathbf{R}^{2}$ que se tome, temos $\|D(r, A)\|>1$, o que implica dizer que a condição $\|D(r ; A)\|<1$ não é condição necessária para estabilidade.

Quando permitimos os retardamentos $r$ e $s$ variar com o tempo, entretanto, a situação não é tão simples. Siqueira [12] mostrou que a equação

$$
x(t)=A x(t-r(t))+B x(t-s(t))
$$


possui uma solução que não tende a' zero quando $t$ tende $a$ infinito.

Ferreira [06] demonstrou o teorema seguinte cuja prova incluiremos em nosso trabalho para maior clareza.

Teorema 2.1: Se $A_{j} A_{k}^{T}=A_{K}^{T} A_{j}$ para $j, k=1,2, \ldots, N$, então o sistema (2.1) é uniformemente assintoticamente estāvel globalmente nos retardamentos, se, e somente se, para a norma

$$
\|D(r, A)\|=\sup _{0 \leq \theta_{k} \leq 2 \pi}\left\|\sum_{k=1}^{N} A_{k} e^{i \theta_{k}}\right\|
$$

tivermos $\|D(x, A)\|<1$

\section{Demonstração:}

$$
\text { Seja } \theta=\left(\theta_{j}, \theta_{2}, \ldots, \theta_{N}\right) \in[0,2 \pi]^{N}, \quad \text { e seja }
$$

$B(\theta)=\sum_{k=1}^{N} A_{k} e^{i \theta_{k}}$. A condição de comutatividade das matrizes $A_{k}$ implica que, para cada $\theta \in[0,2 \pi]^{\dot{N}} \quad a$ matriz $B(\theta)$ é normal então, pelo Lema 1.1 do capítulo I, $\|B(\theta)\|=\gamma(B(\theta))$, onde $\|\cdot\|$ é a norma espectral, e $\gamma($.$) è o raio espectral. Portanto, pelo Teorema 1.3$ do capitulo I, concluímos a demonstração.

Vamos agora, utilizando a condição de comutatividade das matrizes $A_{k}$ demonstrar o resultado principal do nosso trabalho. 
- Consideremos o sistema

(2.4) $\left\{\begin{array}{l}y(t) \doteq \sum_{k=1}^{N} A_{k} y\left(t-r_{k}(t)\right) ; \quad t \geq 0 \\ y(t)=\varphi(t) ;:-r \leq t \leq 0\end{array}\right.$

onde $r_{k}:[0, \infty) \rightarrow[0, \infty)$ são funções contīnuas $r_{k}(0)=r_{k}$; $r_{k}(t) \leq t+r_{k}, \forall t \in[0, \infty), k=1,2, \ldots, N, \quad r_{k}$ e $A_{k}$ como definidos no sistema (2.1), com $A_{k} A_{j}^{T}=A_{j}^{T} A_{k}$ para todo $j, k=1,2, \ldots, N$

Vamos definir

$$
r(t)= \begin{cases}\max \left\{r_{k}(t)\right\} & t \geq 0 \\ r=\max _{l \leq k \leq N}\left\{r_{k}\right\} ; & -r \leq t<0\end{cases}
$$

Teorema 2.2. Suponhamos $A_{k}, r_{k}(t) ; k=1,2, \ldots, N$ e $r(t)$ como acima e suponhamos ainda que $\lim _{t \rightarrow \infty}(t-r(t))=+\infty$. Então, para todo $\varphi \in C_{D}=\left\{\varphi \in C: \varphi(0)={\underset{j}{k=1}}_{\sum_{k}}^{N} A_{k} \varphi\left(-r_{k}\right)\right\}$, sistema (2.4) tem uma única solução $y$ com $y(t) \rightarrow 0$ quando $t \rightarrow \infty$.

\section{Prova:}

Existência e Unicidade:

Seja $M=\left\{y \in C_{H}\left([-r ; \infty), \mathbf{R}^{n}\right): y(t)=\varphi(t),-r \leq t \leq 0\right\}$. on- 
de $C_{H}\left([-r, \infty), \mathbf{R}^{n}\right)=\left\{y \in C\left([-r, \infty), \mathbf{R}^{n}\right):\|\ddot{y}\| \leq H\right\}$, e seja F uma transformação definida em $M_{\text {; }}$ dada por:

$$
\left\{\begin{array}{l}
(F y)(t)=\sum_{k=1}^{N} A_{k} Y\left(t-r_{k}(t)\right) ; \quad t \geq 0 \\
(F y)(t)=y(t) ;-r \leq t \leq 0
\end{array}\right.
$$

F è uma contração, pois

$\left|\left(F Y_{1}\right)(t)-\left(F Y_{2}\right)(t)\right|=\left|\sum_{k=1}^{N} A_{k} Y_{1}\left(t-r_{k}(t)\right)-\sum_{k=1}^{N} A_{k} Y_{2}\left(t-r_{k}(t)\right)\right|=$ $=\left|\sum_{k=1}^{N} A_{k}\left[y_{1}-y_{2}\right]\left(t-r_{k}(t)\right)\right| \leq\|D(r, A)\|\left\|y_{1}-y_{2}\right\|$. Então, pe10 Teorema do Ponto Fixo de Banach, (2.4) tem soiução única.

\section{Estabilidade:}

Façamos a seguinte mudança de variāvel:

$$
y(t)=x(t) \exp (t-r(t)), \text { onde } \exp (\theta)=e^{\theta}
$$

Assim,

$$
y\left(t-r_{k}(t)\right)=x\left(t-r_{k}(t)\right) \exp \left[t-r_{k}(t)-r\left(t-r_{k}(t)\right)\right],
$$

e o sistema $(2.4)$ é equivalente a

$$
\left\{\begin{array}{l}
x(t)=\sum_{k=1}^{N} A_{k} \exp \left[r(t)-r_{k}(t)-r\left(t-r_{k}(t)\right)\right] x\left(t-\dot{r}_{k}(t)\right) ; t \geq 0 \\
x(t)=e^{r(0)-t} \varphi(t) ; \quad-r \leq t \leq 0
\end{array}\right.
$$




$$
\text { Seja } \quad c_{p}=c([-r, \infty), p(t)) \quad \text { com } p(t)=\exp (t-r(t)) \text {. }
$$

$$
\text { Seja } \tilde{M}=\left\{x \in c_{p}: x(t)=e^{r(0)-t} \psi(t) ;-r \leq t \leq 0\right\}
$$

e seja $F$, a transformação contínua em $\tilde{M}$, definida por:

$$
\left\{\begin{array}{l}
(F x)(t)=\sum_{k=1}^{N} A_{k} \exp \left[r(t)-r_{k}(t)-r\left(t-r_{k}(t)\right)\right] x\left(t-r_{k}(t)\right) ; t \geq 0 \\
(F x)(t)=x(t) ;-r \leq t \leq 0
\end{array}\right.
$$

$$
\text { Para todo } x \in \tilde{M} \text { e } t \geq 0 \text {, temos }
$$

$|(F x)(t)| \exp (t-r(t))=$

$=\left|\sum_{k=1}^{N} A_{k} \exp \left[t-r_{k}(t)-r\left(t-r_{k}(t)\right)\right] x\left(t-r_{k}(t)\right)\right| \leq$

$\leq\|D(x, A)\|\|x\|$

$$
\text { Como }\|D(r, A)\|<1 \text { e }(F x)(t)=x(t) ; \quad-r \leq t \leq 0
$$

tem-se

$\|F \times\| \leq\|x\|, \forall x \in \tilde{M}$.

Logo, $F$ aplica $\tilde{M}$ em si mesmo e leva a bola $\mathrm{K}=\mathrm{K}(0, \delta)$ nela mesmo, $\forall \delta \in(0, \infty)$.

$$
\text { Agora, } \forall x, y \in k \quad t \geq 0
$$

$|(F x)(t)-(F y)(t)| \exp (t-r(t))=$

$=\left|\sum_{k=1}^{N} A_{k} \exp \left[r(t)-r_{k}(t)-r\left(t-r_{k}(t)\right)\right](x-y)\left(t-r_{k}(t)\right) \exp (t-r(t))\right| \leq$ 


$$
\begin{aligned}
& =\left|\sum_{k=1}^{N} A_{k} \exp (\Psi(t)) x\left(t-r_{k}(t)\right)-\sum_{k=1}^{N} A_{k} \exp (\Psi(s)) x\left(s-r_{k}(s)\right)\right|= \\
& =\mid \sum_{k=1}^{N} A_{k}\left[\exp (\Psi(t)) x\left(t-r_{k}(t)\right)-\exp (\psi(s)) \times\left(s-r_{k}(s)\right)\right] \leq \\
& \leq\|D(r, A)\| \sup \{|\exp (t-r(t)) x(t)-\exp (s-r(s)) x(s)|: t, s \in(0, \Gamma),|t-s| \leq \tau\} \\
& \quad \therefore \quad t \quad \sup _{\tau}\left\{\left(t-r_{k}(t)\right)-\left(s-r_{k}(s)\right)|; \quad t, s \in(0, \Gamma),| t-s \mid \leq \varepsilon\right\}
\end{aligned}
$$$$
\text { Logo, }
$$$$
\omega_{0}^{F}(F x) \leq\|D(r, A)\| \omega_{0}^{\Gamma}(\dot{X}), \forall x c k: \text { fixo. }
$$$$
\text { Portanto, }
$$$$
\omega_{0}(F X) \leq \| D\left(r, A \| \omega_{0}(X) ; \forall x C K:\right. \text { fixo. }
$$

$$
\begin{aligned}
& \text { Combinando }(2.7) \text { e }(2.8) \text {, temos: } \\
& \mu_{0}(F X) \leq\|D(r, A)\| \mu_{o}(X), \forall x \text { C } \text { : fixo. }
\end{aligned}
$$

Assim, temos que $F$ è $\mu_{0}$-contração, e pelo Teorema 1.5 do capítulo $I$, o sistema (2.5) tem um ponto fixo $\mathrm{x} \in \mathrm{C}_{\mathrm{p}}, \cdot \operatorname{com}:$

$$
\begin{aligned}
& \text { (2.9) } \lim _{t \rightarrow \infty} x(t) p(t)=0,: \text { e como } \\
& x(t)=\exp [-(t-r(t))] y(t), \quad \text { tem-se }
\end{aligned}
$$


$\lim _{t \rightarrow \infty} x(t) p(t)=\lim _{t \rightarrow \infty} \exp [-(t-r(t))] y(t) \exp (t-r(t))=\lim _{t \rightarrow \infty} y(t)$ Portanto, usando (2.9), tem-se $\lim _{t \rightarrow \infty} \mathrm{y}(t)=0$, onde y è a solução do sistema (2.4). 
Bibliografia

[01] AVELLAR, C.E. \& HALE, J.K. On the zeros of exponencial polynomials. J. Math. Anal. Appl. 73(1980), 434-452 .

[02] AVELLAR, C.E. Effects of the Delay in Differential Difference Equations, Thesis, Brown University, June, 1979.

[03] BANÃS, J.; HAJNOSZ, A. \& WEDRYCHOWICZ, C. On Existence and asymptotic behavior of solutions of some functional equations. Funkcial. Ekvac 25(1982), 257$-267$.

[04] BANÃ̃, J.; \& GOEBEL, K. Measures of Noncompactness in Banach Spaces. Marcel Dekker, Inc., 1980.

[05] CRUZ, M.A. \& HALE, J.K. stability of functional differential equations of neutral type. J. Differential Equs. $7(1970), 334-355$.

[06] FERREIRA, J.M. Effects of commensurable delays in the stability of difference equations. Royal Society "of Edinburgh $100 \mathrm{~A}, 191-199$, (1985).

[07] HALE, J.K. A class of neutral equations with the fixedpoint property. Proc. Nac. Acad. Sci., USA. 67 (1970), $136-137$. 
[08] HALE, J.K. Theory of Functional Differential Equations. Springer-Verlag, New York, (1977).

[09] HALE, J.K. \& MEYER, K.R. A class of functional equations of neutral type. Men. Amer. Math. Soc., 76 (1967).

[10] HENRY, D. Linear autonomous neutral functional differential equations. J. Differential Equs. 15 (1974), 106-128.

[1i] MELVIN, W.R. Stability properties of functional differential equations. J. Math. Anal. Appl. 48(1974), $749-763$.

[12] SIQUEIRA, S.A. Equação de Diferença com Retardamento Dependendo do Tempo. Dissertação, ICMSC-USP, ' 1984 .

[13] SILKOWSKI, R.A. Star-Shapead regions of stability in hereditary systems. $\underline{\mathrm{Ph}}$. D. Thesis, Brow University, Providence, R.I., June 1976.

[14] VARGA, R.S. Matrix Iterative Analysis Prentice-Hall, Inc., Englewood Cliffs, New Jersey 1962. 